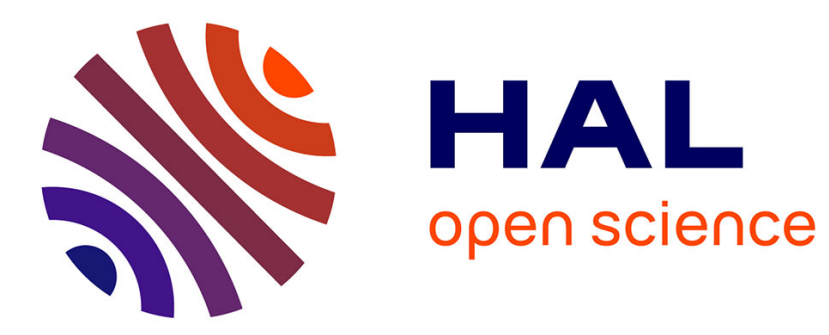

\title{
Multiangle retrodirective cascaded metasurface
}

Mohammed Kalaagi, Divitha Seetharamdoo

\section{To cite this version:}

Mohammed Kalaagi, Divitha Seetharamdoo. Multiangle retrodirective cascaded metasurface. Journal of Applied Physics, 2019, 126, p. 104901. 10.1063/1.5095147 . hal-02924923

\section{HAL Id: hal-02924923 \\ https://hal.science/hal-02924923}

Submitted on 28 Aug 2020

HAL is a multi-disciplinary open access archive for the deposit and dissemination of scientific research documents, whether they are published or not. The documents may come from teaching and research institutions in France or abroad, or from public or private research centers.
L'archive ouverte pluridisciplinaire HAL, est destinée au dépôt et à la diffusion de documents scientifiques de niveau recherche, publiés ou non, émanant des établissements d'enseignement et de recherche français ou étrangers, des laboratoires publics ou privés. 


\title{
Multi Angle Retrodirective Cascaded Metasurface
}

\author{
Mohammed Kalaagi ${ }^{1, \text { a) }}$ and Divitha Seetharamdoo ${ }^{1,}$ b) \\ ${ }^{1}$ Univ. Lille Nord de France, IFSTTAR, COSYS, LEOST, F-59650 Villeneuve D ascq, \\ France
}

(Dated: 24 July 2020)

In this paper a cascaded retrodirective metasurface is designed and demonstrated to operate simultaneously at a wide range of incident angles between $-30^{\circ}$ to $-10^{\circ}$ and $10^{\circ}$ to $30^{\circ}$. It is based on the design of several retrodirective super-cells following the generalized Snell's law of reflection, where each super-cell is designed to redirect an incoming wave back in the same direction with high efficiency. This metasurface is a very good candidate as a retroreflector for radar cross-section enhancement of targets with poor backscattering. Retrodirective topologies have been a subject of interest and several engineered topologies exist such as the corner dihedral. Despite their good performances at a range of incident angles, their 3-dimensional bulky structure make them hard to implement for different applications and they do not address extreme incident angles. The metasurface proposed can be a complementary solution to existing topologies for addressing extreme oblique incident angles while being more compact due to its two dimensional (2D) subwavelength structure design. The monostatic RCS performance of the designed metasurface of dimensions $8.163 \mathrm{~cm} \times 56.23 \mathrm{~cm}$ has been compared to that of a conventional corner dihedral of dimensions $8 \mathrm{~cm} \times 15.5 \mathrm{~cm} \times$ $7.75 \mathrm{~cm}$, a gain up to $50 \mathrm{~dB}$ of monostatic radar cross section (RCS) in the ranges $-30^{\circ}$ to $-20^{\circ}$ and $20^{\circ}$ to $30^{\circ}$ was obtained. Comparable performances are observed in the ranges $-20^{\circ}$ to $-10^{\circ}$ and $10^{\circ}$ to $20^{\circ}$ between the designed metasurface and the corner dihedral. Experimental results are shown to be in good agreement with simulation results.

Keywords: metasurfaces, retrodirectivity, radar cross-section enhancement, gradient index metamaterial

\section{INTRODUCTION}

Metasurfaces $^{1}$ are the subject of intensive research nowadays for their applicative potential and the generality of their design approach ${ }^{2,3}$. They are characterized by their 2D subwavelength structure and their ability to control the wavefront of an electromagnetic wave ${ }^{4,5,6}$ with perfect, abnormal and achromatic reflections ${ }^{7,8,9}$. One of the common metasurfaces are phase-gradient surfaces 10 designed to tailor or manipulate a reflected and transmitted electromagnetic wave ${ }^{11,7}$. Other applications have been introduced recently for metasurfaces including polarization conversion ${ }^{12}$, antennas broadband diffusion of terahertz waves ${ }^{13}$, hologram ${ }^{14}$, Huygens' metasurfaces 8 , multi-functional metasurfaces 15,9 , transformation of propagating waves into surface waves ${ }^{2,16}$ and total control or beam steering ${ }^{17}$ metasurfaces at optical and microwave frequencies $^{18}$.

The control of an electromagnetic wave for retrodirective reflection has gained lot of interest recently for various applications. Retro-reflection ${ }^{19}$, can be obtained from diffraction in blazed grating $20,21,22$, such as in a Littrow configuration ${ }^{23}$. More recently, flat metaldielectric surfaces for retro-reflection or more generally for anomalous reflection ${ }^{2}$, have been designed based on generalized phase law $^{24}$ and reflect-array approach ${ }^{25}$. V.S Asadchy et. $a l^{26}$ have proposed surface impedance modulation techniques for efficiency optimization . If one considers the applicative potential of such these retrore-

\footnotetext{
a) Electronic mail: mohammed.kalaagi@ifsttar.fr

b) Electronic mail: divitha.seetharamdoo@ifsttar.fr
}

flective metasurfaces, the control of an electromagnetic wave in a retrodirective manner can be an important feature for the design of radar targets in order to enhance their radar cross section (RCS) ${ }^{27}$ particularly when these targets have poor inherent backscattering properties. This phenomenon is generally achieved using engineered topologies such as corner dihedrals ${ }^{28}$ and Van Atta array technologies ${ }^{28}$. Although these topologies can perform well for retrodirectivity at specific range of incident angles, they can be bulky and hard to implement for applications involving automotive radar applications where road user such as pedestrians and cyclists need to be equipped to increase their probability of detection. It is therefore interesting to provide equivalent retroreflective features with more compact topologies and less complex implementations.

In this paper we are interested in investigating the potential of metasurfaces and their performances, in terms of retroreflection for multiple incident angles such that they can be used for the enhancement of radar crosssection of targets. It has been shown for the design of phase gradient metasurfaces following the generalized Snell's law ${ }^{24}$ of reflection, that an engineered metasurface design can manipulate an electromagnetic wave in a desired anomalous direction. In the case of retrodirective control of an electromagnetic wave using engineered flat plate metasurfaces, the starting point is the conventional Snell's law of physical optics where retrodirectivity can be obtained at normal incidence, reflecting back from a mirror or a flat mettalic plate. If oblique incidence angles are to be considered, it can be shown that a phasegradient designed surface can control the electromagnetic wave, to reflect it back in the same direction of incidence even at extreme angles. 
A cascaded multiple incident angle retrodirective metasurface (C-MIARM) is proposed. The first design step consists in designing metasurfaces operating at single incident angles for the same operating frequency following the generalized phase law of reflection ${ }^{24}$ and finally optimizing their association without any loss in performance. The angles of incidence considered are $\pm 30^{\circ}$, $\pm 25^{\circ}, \pm 20^{\circ}, \pm 15^{\circ}, \pm 10^{\circ}$. We have observed that with an increase in the periodicity of the super-cell design of $2 \lambda$ or higher, the metasurface loses its symmetrical response with respect to retrodirectivity for the given desired angle, i.e. retroreflection is no longer obtained identically for negative and positive values of the desired angle of incidence. A new structure is introduced for the super-cell design at $10^{\circ}$ and $15^{\circ}$ to recover the loss in the symmetrical direction and maintain the performance of the C-MIARM. The radar cross-section of the C-MIARM is determined both numerically and experimentally. For comparison purposes, a conventional corner dihedral, well known for its retrodirective properties is used in the range between $-20^{\circ}$ and $20^{\circ}$. The performances of the proposed C-MIARM and the corner dihedral are compared in the angular ranges $-30^{\circ}$ to $-10^{\circ}$ and $10^{\circ}$ to $30^{\circ}$.

The paper is organized as follows. Section II defines the general principle of operation for the design of retrodirective metasurfaces following the generalized phase law of reflection as well as the design methodology for the design of a lossless retrodirective super-cell at a specific angle of incidence. In Section III the design of various metasurfaces is given for various angles and periodicities where different performances have been shown. Section IV the design of the C-MIARM is presented. The C-MIARM has been compared to a conventional corner dihedral where their performances have been compared with respect to monostatic RCS enhancement. In Section $\mathrm{V}$ measurements have been performed for a metasurface design at $30^{\circ}$ and the C-MIARM, where it has been shown that the results are in good agreement compared to that of the simulation results.

\section{GENERAL PRINCIPLE OF OPERATION FOR THE DESIGN OF A RETRODIRECTIVE GRADIENT METASURFACE}

Gradient metasurfaces ${ }^{2}$ are well known for their potential of manipulating and controlling of an electromagnetic wave, such as beam steering and anomalous reflection. Retrodirectivity has been an interesting property for metasurfaces, which is defined by reflecting an incoming wave back in the same direction of incidence ${ }^{26}$. The idea of retrodirectivity comes form the concept of the conventional Snell's law of reflection when a wave is reflected from a mirror or metallic surface at normal incidence. In case of a retrodirective designed gradient metasurface, the wave is reflected back from a virtual surface(mirror) which is tilted by an angle $\theta$ as shown in Fig. 1, this shows that after introducing a phase gradient $\delta$ between unit cells, the wave is reflected back from a virtual surface where the initial reference is tilted by an angle $\theta_{i}$, re-directing back in the same direction similar to that of the conventional case for a mirror or flat plate at normal incidence.

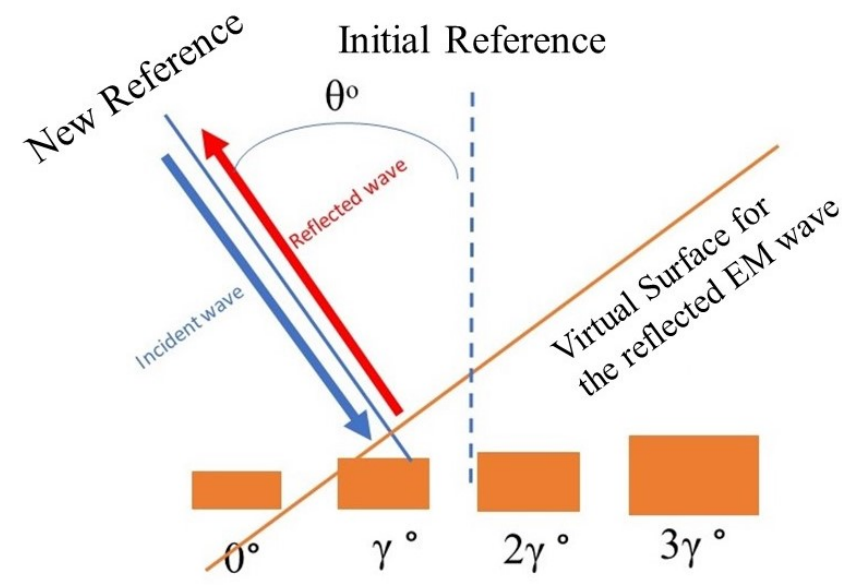

FIG. 1. An incident wave reflecting back from an engineered gradient metasurface in the same direction of incidence

\section{A. Generalized Phase Law of Reflection}

The design of a gradient metasurface is based on the generalized phase law of reflection ${ }^{24}$, where an additional term (the phase gradient) is given with respect to the conventional Snell's law as shown in Eq. (1). This is consistent with the well-known grating equation. ${ }^{23}$

$$
\sin \theta_{r}-\sin \theta_{i}=\frac{\lambda_{0}}{2 \pi} \frac{\partial \phi}{\partial x},
$$

$\theta_{r}$ is the angle of reflection, $\lambda_{0}$ the free space wavelength and $\partial \phi / \partial x$ is the phase gradient.

Thus, by introducing a phase gradient between scatterers on top of an engineered metasurface design, an electromagnetic wave is reflected to a desired anomalous direction. In the case for a retrodirective metasurface de$\operatorname{sign} \theta_{r}$ is said to be equal to $-\theta_{i}$. The periodicity or the dimension of the super-cell design is determined by the integration of Eq. (1). from 0 to $2 \pi$ in order to control the whole wavefront of the propagating electromagnetic wave.

$$
L_{x}=\frac{\lambda_{0}}{2 \sin \theta_{i}}
$$

Eq.(2). defines the periodicity (dimension of the supercell) for a retrodirective super-cell at a given angle of incidence.

\section{B. Design of a Retrodirective Super-Cell}

Following the generalized phase law and after determining the periodicity for a retrodirective super-cell $\mathrm{L}_{x}$, the super-cell is then divided into $\mathrm{N}$ unit cells with a phase gradient $d \phi / d x$ between the unit cell scatterers as shown in Fig. 2. 


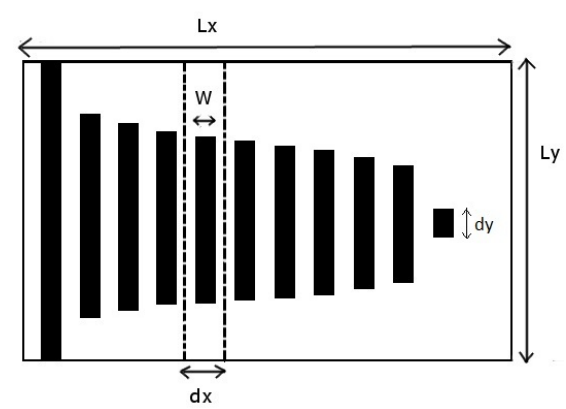

FIG. 2. Super cell divided into subwavelength unit cells of dimensions $L_{x}$ and $L_{y}$

In this case, strip lines metallic patches are used as a unit cell for their subwavelength structure and to ensure low periodicity between the scatterers giving rise to a magnetic coupling for high efficient performance ${ }^{2}$. The unit cells are tuned to obtain a progressive phase shift covering the range of the phase of the reflection phase coefficient for the control of the wavefront. The phase gradient $^{1}$ is given in Eq.(3).

$$
\phi_{N+1}-\phi_{N}=2 K_{0} d_{x} \sin \theta_{i}
$$

$d_{x}$ refers to the periodicity of the unit cell which is defined as $d_{x}=\mathrm{L}_{x} / \mathrm{N}$ and $K_{0}$ is the wave number. In the case of retrodirectivity Eq.(2). is derived into Eq.(3). to determine the phase gradient for a retrodirective supercell which is given in Eq.(4).

$$
\phi_{N+1}-\phi_{N}=\frac{2 \pi}{N}
$$

The phase gradient in Eq.(4) is shown to be dependent on the number of subwavelength unit-cells $N$. The dimensions of the unit cells are determined following the reflection phase coefficient and Eq.(4). The reflection phase coefficient is calculated using Floquet harmonic boundaries Fig.3. for a unit cell of periodicity $d_{x}$ and length $\mathrm{L}_{y}$, with the strip-line of width $\mathrm{w}$ and length $d_{y}$. In this case an example is given for a design at $\theta=30^{\circ}$ and $\lambda$ $=20 \mathrm{~mm}$, with $N=12, w=0.71 \mathrm{~mm}$ and $\mathrm{L}_{y}=\lambda / 2$. The metallic strip-lines are printed on a grounded substrate of permitivitty $\epsilon=2.2, \tan (\delta)=1 \times 10^{-3}$ and thickness $1.57 \mathrm{~mm}$.

Following the curve of the reflection phase coefficient given in Fig.3. and Eq.(4), the phase gradient is given as $d \phi / d x$ equal to $30^{\circ}$. The dimensions of the strip lines are given in Table 1. Eleven strip lines are given in this case due to the fact that the phase of the last strip line corresponds to zero.

\section{Lossless Reflection Condition}

In order to ensure a high efficient retrodirective wave, the lossless reflection condition should be validated. It has been shown ${ }^{29}$ that in order to obtain a high efficient

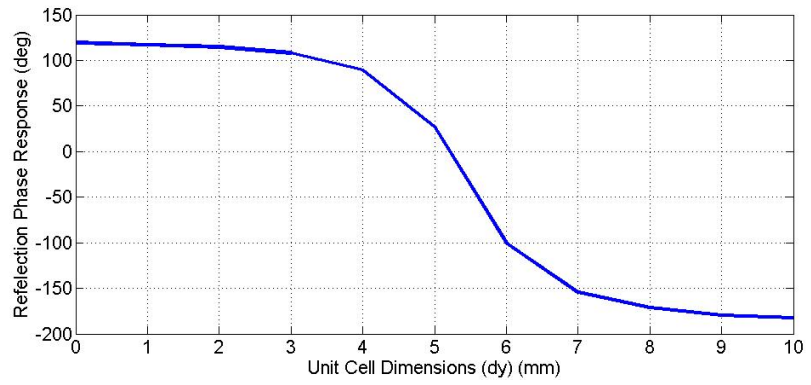

FIG. 3. Phase of the reflection coefficient for a TM polarized wave at $14.7 \mathrm{GHz}$ with respect to the dimensions of the striplines $d_{y}$.

TABLE I. Reflection phases with respect to the dimensions of the strip-lines $d_{y}$ with a phase gradient $\phi_{N}$ equal to $30^{\circ}$

\begin{tabular}{cc}
\hline \hline Phase Coefficient (degrees) & Dimensions $d_{y}(\mathrm{~mm})$ \\
\hline-182.6812 & 10.2 \\
-152.6812 & 7.1 \\
-122.6812 & 6.5 \\
-92.6812 & 6.06 \\
-62.6812 & 5.82 \\
-32.6812 & 5.6 \\
-2.6812 & 5.34 \\
27.3188 & 5.1 \\
57.3188 & 4.6 \\
87.3188 & 4.12 \\
117.3188 & 1 \\
147.3188 & 0 \\
\hline
\end{tabular}

reflected signal, the normal component of the Poynting vector should be less than or equal to zero $\mathrm{P}_{n} \leq 0$ which is satisfied when the reflected electric field $E_{r}$ is equal to that of the incident electric field $E_{i}\left(\mathrm{E}_{r}=\mathrm{E}_{i}\right) . \mathrm{P}_{n}$ is defined as follows:

$$
\mathbf{P}_{n}=\frac{E_{i}^{2}}{2 \eta_{1}}\left(\cos \theta_{r}-\cos \theta_{i}\right)\left[1+\cos \phi_{r}(z)\right]
$$

where $\phi_{r}$ defines the reflection phase. Taking the example of the design at $30^{\circ}, \mathrm{P}_{n}$ has been calculated as shown in Fig. 4.

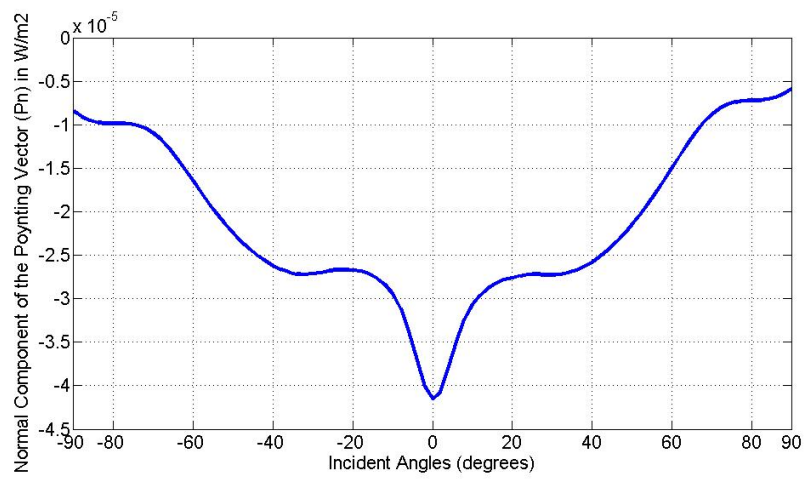

FIG. 4. The normal component of the Poynting vector with respect to $\theta_{i}$ for a metasurface design at $30^{\circ}$.

The result shows that $\mathrm{P}_{n}$ is less than zero for all incident angles, thus validating the condition of Eq.(5). 


\section{DESIGN OF RETRODIRECTIVE METASURFACES FOR SEVERAL ANGLES OF INCIDENCE}

Following the design procedure and the general principle of operation for that at $30^{\circ}$, different metasurfaces are designed at $25^{\circ}, 20^{\circ}, 15^{\circ}$ and $10^{\circ}$ each with a different periodicity with respect to $\lambda$.

The study of the monostatic RCS performance for each design is given and compared to that of a flat metallic plate, for efficiency studies and having the metallic flat plate as a reference which is well known for its performance for retrodirectivity at normal incidence. For the dimensions of the super-cell designs and the phases of the reflection coefficient for each incident angle case, refer to the supplementary material of the journal.

\section{A. Monostatic RCS Performance for a Retrodirective Metasurface at $30^{\circ}$}

After the design of a retrodirective super-cell following the general principle of operation, an array periodic structure of $7 \times 9$ super-cells is printed on top of a Teflon substrate of permittivity $\epsilon=2.2$ as shown in Fig. 5 .

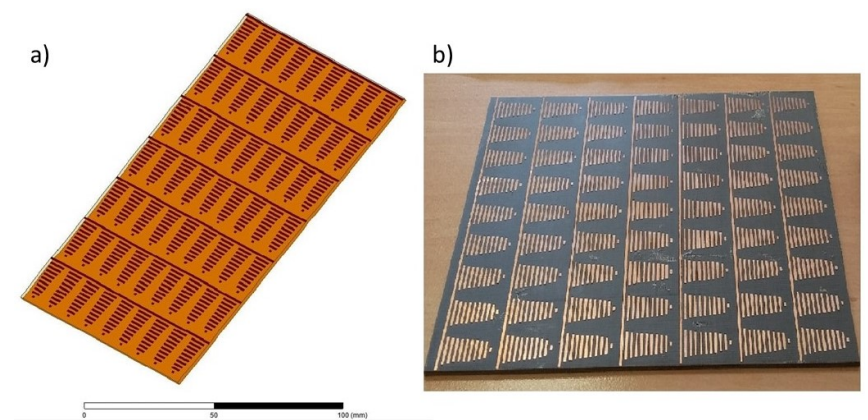

FIG. 5. a) Metasurface design at $30^{\circ}$ Using Ansys HFSS, b) Metasurface prototype of $7 \times 9$ super-cells printed on a Teflon substrate of dimensions $14.3 \mathrm{~cm} \times 9.2 \mathrm{~cm}$ and thickness $1.57 \mathrm{~mm}$.

For $\theta=30^{\circ}$ the periodicity $\mathrm{L}_{x}$ is equal to $\lambda$ according to Eq.(2). The monostatic RCS for the design is given in Fig. (6) and compared to that of a conventional metallic flat plate.

The result in Fig. 6. shows a high value of monostatic RCS at the desired angles as well as at $-30^{\circ}$ and normal incidence, the symmetrical response of the metasurface is expected, this is due to the similar response in both directions of the phase of the reflection coefficient from $117^{\circ}$ to $-182.68^{\circ}$ and from $-182.68^{\circ}$ to $117^{\circ}$ as shown in Fig.3 .

\section{B. Monostatic RCS Performance for Retrodirective Metasurface designs at $25^{\circ}$ and $20^{\circ}$}

The same procedure is followed for the designs at $25^{\circ}$ and $20^{\circ}$ given to that at $30^{\circ}$. The periodicity $\mathrm{L}_{x}$ is $1.18 \lambda$ and $1.462 \lambda$ at $25^{\circ}$ and $20^{\circ}$ respectively. The monostatic RCS of the given designs is shown in Fig. 7 and 8 .

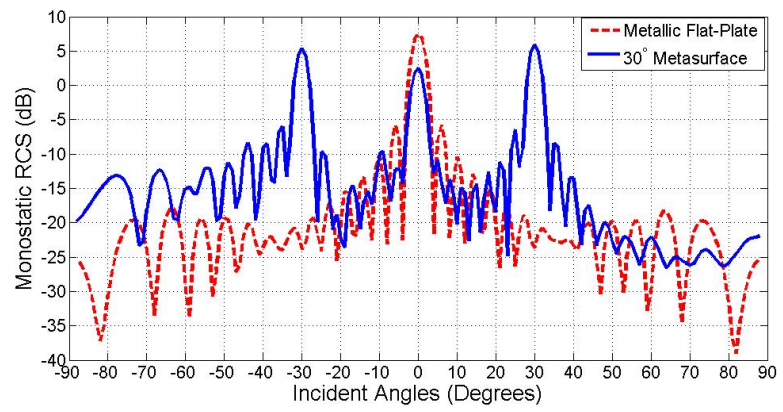

FIG. 6. Monostatic RCS (dB) with respect to the incident angles (degrees) for a $30^{\circ}$ metasurface design of $7 \times 9$ super cells and dimensions $14.3 \mathrm{~cm} \times 9.2 \mathrm{~cm}$ compared to that of a metallic flat plate of similar dimensions.

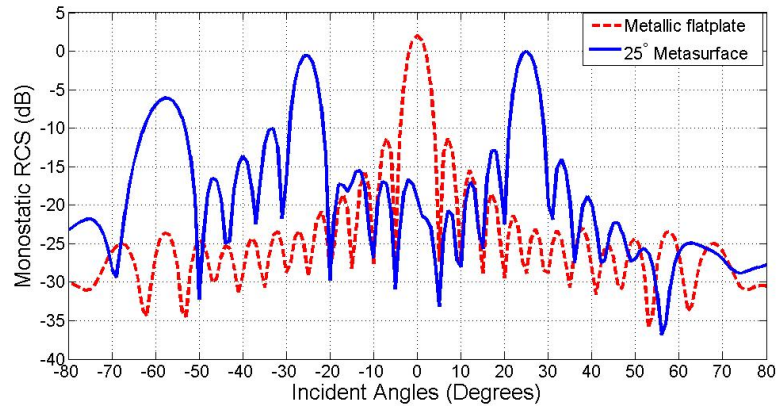

FIG. 7. Monostatic RCS (dB) with respect to the incident angles (degrees) for a $25^{\circ}$ metasurface design of $6 \times 5$ supercells and dimensions $6.1 \mathrm{~cm} \times 12 \mathrm{~cm}$ compared to that of a Flat metallic plate of similar dimensions.

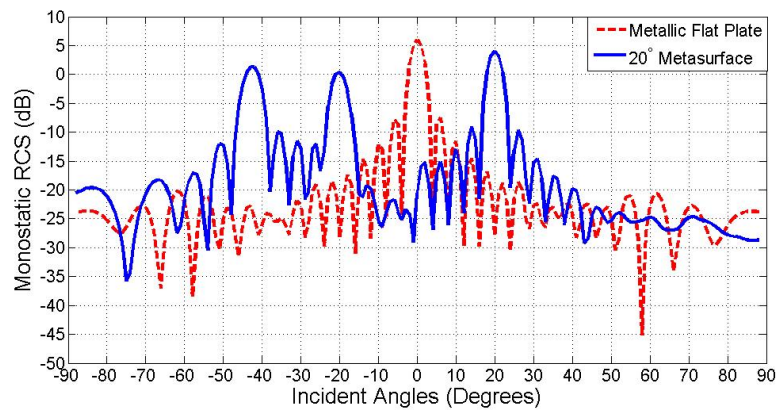

FIG. 8. Monostatic RCS (dB) with respect to the incident angles (degrees) for a $20^{\circ}$ metasurface design of $7 \times 5$ supercells and dimensions $7.1 \mathrm{~cm} \times 15 \mathrm{~cm}$ compared to that of a flat metallic plate of similar dimensions.

In Fig. 7. a high value of monostatic RCS is given at $25^{\circ}$ as well as at $-25^{\circ}$ due to the symmetrical response of the metasurface design, whereas an absence at normal incidence compared to that for the $30^{\circ}$ design which is the result of a periodicity $\mathrm{L}_{x}>\lambda$. In this case higher orders of diffraction are present $\left(\right.$ at $-58^{\circ}$ ) as the periodicity increases higher than $\lambda$, as expected according to Floquet-Bloch analysis ${ }^{30}$

Fig. 8. shows the result of the monostatic RCS for the design at $20^{\circ}$ giving a high value at $20^{\circ}$ and $-20^{\circ}$ with a diffracted order at $-44^{\circ}$ with a significant value in terms 
of retrodirectivity.

\section{Monostatic RCS Performance for Retrodirective Metasurface designs at $15^{\circ}$ and $10^{\circ}$}

The design of a retrodirective metasurface is given in this case where the periodicity $\mathrm{L}_{x}$ is equal to $2 \lambda$ and $2.88 \lambda$ at $15^{\circ}$ and $10^{\circ}$ respectively. The monostatic RCS of the given designs is shown in Fig. 9 and 10.

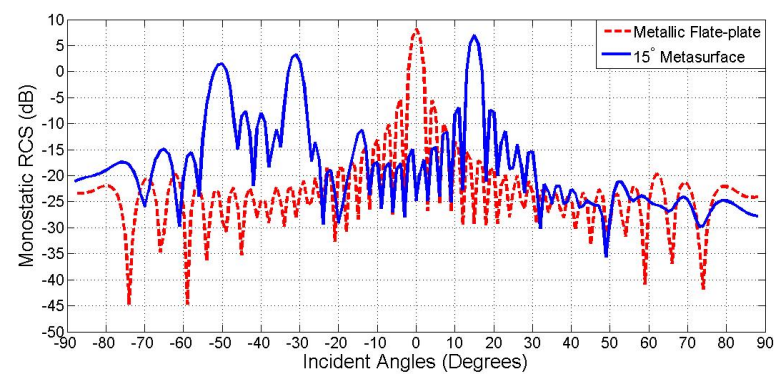

FIG. 9. Monostatic RCS (dB) with respect to the incident angles (degrees) for a $15^{\circ}$ metasurface design of $7 \times 5$ supercells and dimensions $7.14 \mathrm{~cm} \times 19.7 \mathrm{~cm}$ compared to that of a metallic flat plate of similar dimensions.

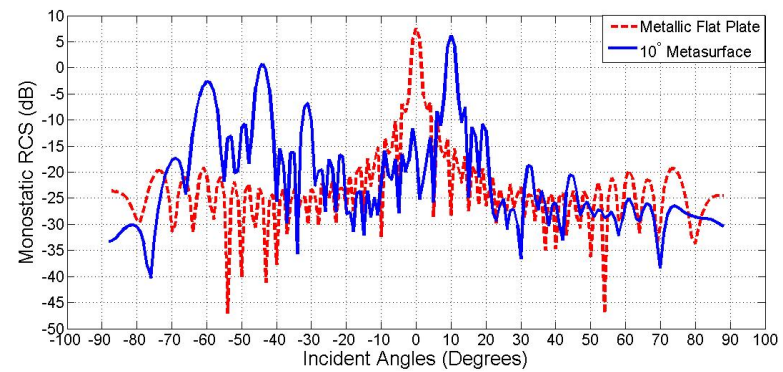

FIG. 10. Monostatic RCS (dB) with respect to the incident angles (degrees) for a $10^{\circ}$ metasurface design of $6 \times 4$ supercells and dimensions $6.12 \mathrm{~cm} \times 23.5 \mathrm{~cm}$ compared to that of a metallic flat plate of similar dimensions.

The result in Fig. 9. shows a high value of monostatic RCS at $15^{\circ}$. It can be shown in this case, that the symmetrical response in the opposite direction at $15^{\circ}$ is lost, although the phase of the reflection coefficient remains with the same response compared to that at $30^{\circ}, 25^{\circ}$ and $20^{\circ}$ (refer to supplementary material). Two diffracted orders are present in this case showing a high value of monostatic RCS at $-32^{\circ}$ and $-50^{\circ}$ as shown in Fig. 9. The symmetrical response in terms of retrodirectivity for the metasurface design is lost due to the high periodicity $\mathrm{L}_{x}$ in this case, being $\geq 2 \lambda$ and the presence of the higher orders of diffraction.

Fig. 10. shows the result of the monostatic RCS for a $10^{\circ}$ designed metasurface with a periodicity $\mathrm{L}_{x}=2.88 \lambda$. A high value of monostatic RCS is present at the desired angle with the absence of retrodirectivity in the opposite direction at $-10^{\circ}$ and two significant values at the diffracted orders at $-44^{\circ}$ and $-60^{\circ}$.

\section{DESIGN OF A MULTIPLE INCIDENT ANGLE RETRODIRECTIVE CASCADED METASURFACE}

The aim is to design a multi-angle retrodirective metasurface at a wide range of incident angles in the range between $-30^{\circ}$ and $30^{\circ}$ with a symmetrical response. The design is based on the superposition of different designed super-cells at various angles $\left(30^{\circ}, 25^{\circ}, 20^{\circ}, 15^{\circ}\right.$ and $10^{\circ}$ ) forming a cascaded metasurface operating simultaneously for multiple incident angles.

\section{A. Metasurfaces with Symmetrical Responses at $-15^{\circ}$ and $-10^{\circ}$ for periodicity greater than $2 \lambda$}

As mentioned before, the metasurface design for a periodicity $\geq 2 \lambda$ shows a loss in the symmetrical response of the metasurface design. Thus, a different design structure is proposed for the metasurfaces at $15^{\circ}$ and $10^{\circ}$ by introducing a metasurface with two opposite directions as shown in Fig. 11. The results for the monostatic RCS for the proposed design structure at $15^{\circ}$ and $10^{\circ}$ are given in Fig. 12 and 13.

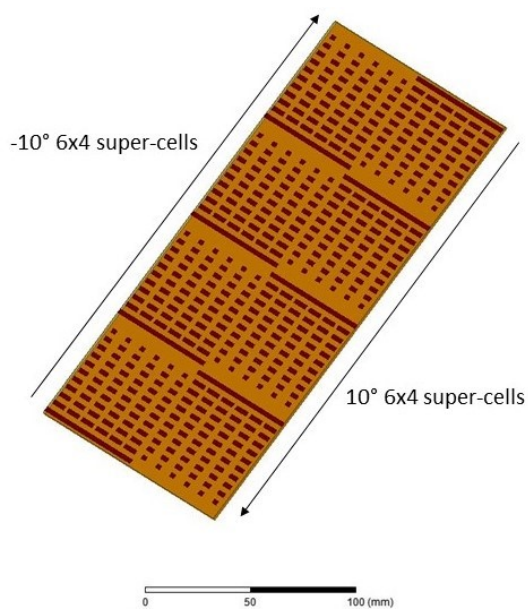

FIG. 11. A metasurface design with two opposite directions structure at $10^{\circ}$ using Ansys HFSS.

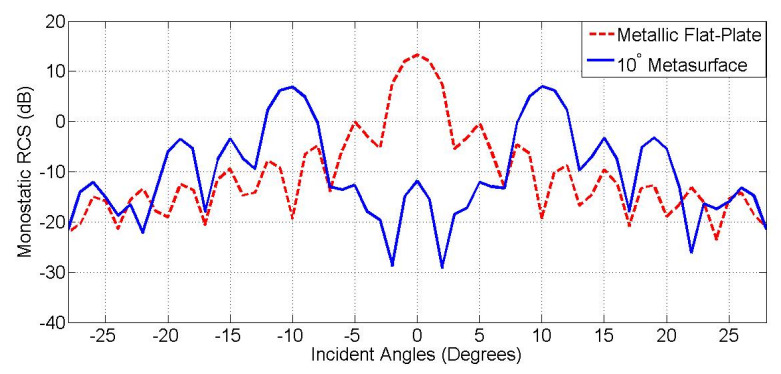

FIG. 12. Monostatic RCS of the $10^{\circ}$ metasurface proposed structure of $12 \times 4$ super-cells(Fig. 11) of dimensions 12.224 $\mathrm{cm} \times 23.5 \mathrm{~cm}$ compared to that of a flat metallic plate of similar dimensions.

Fig. 12 and 13 show the comparison between the proposed metasurface designs at $10^{\circ}$ and $15^{\circ}$ to that of a flat 


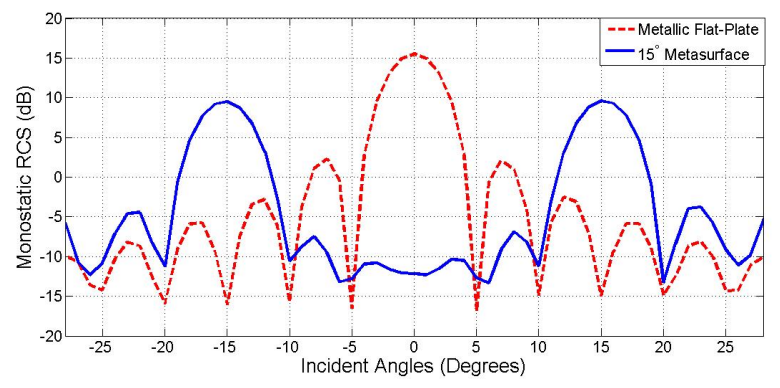

FIG. 13. Monostatic RCS of the $15^{\circ}$ metasurface proposed structure of $14 \times 5$ super-cells of dimensions $14.28 \mathrm{~cm} \times 19.7$ $\mathrm{cm}$ compared to that of a flat metallic plate of similar dimensions.

metallic plate respectively. The results of the monostatic RCS in Fig. 12 and 13, show a recovery of the loss in the symmetrical directions at $-10^{\circ}$ and $-15^{\circ}$ for the desired angles of incidence using the proposed structure design for both cases with lower efficiency with respect to that of the flat metallic plate, compared to that of the metasurfaces which show a high value only for the angles at $10^{\circ}$ and $15^{\circ}$, but regaining the loss of the symmetrical response of the metasurface (lower ranges in this case of incident angles have been used from $-28^{\circ}$ to $28^{\circ}$ for the simplicity of the computational time analysis and highlight the concept) .

\section{B. Cascaded Multiple Incident Angle Retrodirective Metasurface (C-MIARM)}

The Cascaded Multiple Incident Angle Retrodirective Metasurface (C-MIARM) is designed after the superposition of the metasurfaces at $30^{\circ}, 25^{\circ}, 20^{\circ}, 15^{\circ}$ and $10^{\circ}$, using the opposite metasurface structure for the designs at $15^{\circ}$ and $10^{\circ}$ as shown in Fig. 14.

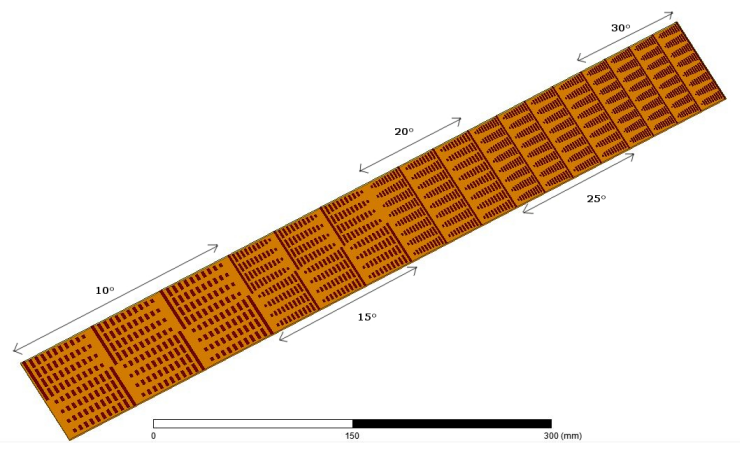

FIG. 14. Cascaded Multiple Incident Angle Retrodirective Metasurface (C-MIARM) with the superposition of $4 \times 830^{\circ}$, $25^{\circ}$ and $20^{\circ}$ super-cells each with $15^{\circ}$ and $10^{\circ}$ metasurfaces using the mirror image form, where the MIARM has an overall dimension of $8.163 \mathrm{~cm} \times 56.23 \mathrm{~cm}$ and substrate of $\epsilon=2.2$ and thickness $1.57 \mathrm{~mm}$.

The dimensions of the C-MIARM are $8.163 \mathrm{~cm} \times 56.23$ $\mathrm{cm}$, the normalized monostatic RCS with respect to the metasurfaces at various angles is given in order to determine the overall performance of the designed metasurface as shown in Fig. 15.

The results have shown a wide range of monostatic RCS with respect to various incident angles in the ranges between $-30^{\circ}$ to $-10^{\circ}$ and from $10^{\circ}$ to $30^{\circ}$. A low value is present in the range between $-10^{\circ}$ to $0^{\circ}$ which can be said to be due to the absence of a metasurface design at $5^{\circ}$ for the simplicity of the numerical analysis, whereas an intermediate value was shown between $0^{\circ}$ and $10^{\circ}$.

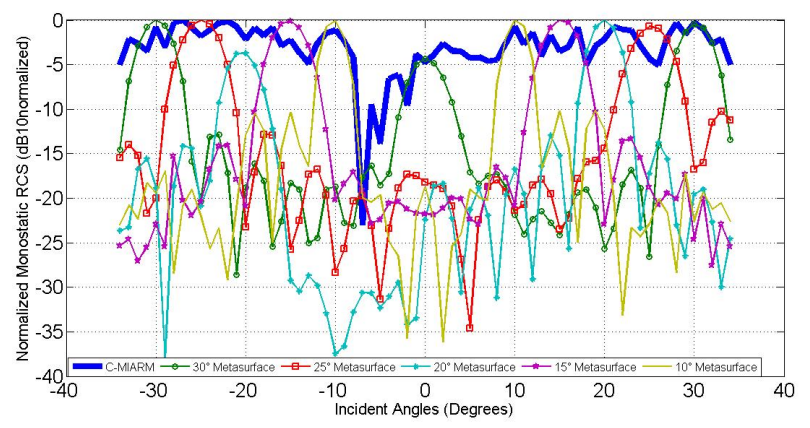

FIG. 15. Normalized monostatic RCS in dB of the C-MIARM with respect to the overall performances of the metasurfaces designed at $30^{\circ}, 25^{\circ}, 20^{\circ}, 15^{\circ}$ and $10^{\circ}$.

\section{Comparison between the C-MIARM and the Conventional Corner Dihedral}

Different topologies of retrodirective reflectors have been shown in the literature including the corner dihedral. The results and performance of the C-MIARM with respect to monostatic RCS is compared to that of the corner dihedral as shown in Fig. 16.

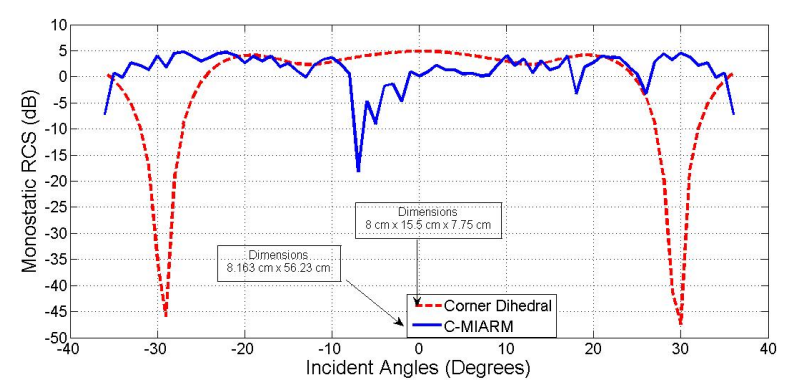

FIG. 16. Monostatic RCS of the C-MIARM of dimensions $8.163 \mathrm{~cm} \times 56.23 \mathrm{~cm}$ compared to that of a conventional corner dihedral of dimensions $8 \mathrm{~cm} \times 15.5 \mathrm{~cm} \times 7.75 \mathrm{~cm}$.

The results in Fig. 16. show a $50 \mathrm{~dB}$ gain of monostatic RCS for the C-MIARM with repect to the corner dihedral of dimensions $8 \mathrm{~cm} \times 15.5 \mathrm{~cm} \times 7.75 \mathrm{~cm}$ in the range between $-30^{\circ}$ to $-20^{\circ}$ and $20^{\circ}$ to $30^{\circ}$, whereas the corner dihedral was limited between $-20^{\circ}$ to $20^{\circ}$ where the CMIARM showed a wide range between $-30^{\circ}$ to $-10^{\circ}$ and $10^{\circ}$ to $30^{\circ}$.

Giving a value up to $5 \mathrm{~dB}$ of monostatic RCS, the CMIARM was able to achieve this value with dimensions 
$8.163 \mathrm{~cm} \times 56.23 \mathrm{~cm}$ compared to that of a 3D structured corner dihedral of dimensions $8 \mathrm{~cm} \times 15.5 \mathrm{~cm} \times 7.75$ $\mathrm{cm}$. The advantage of the C-MIARM is having a $2 \mathrm{D}$ structure making it easy for implementation for different application and addressing extreme incident angles, and can be a complementary solution for such topologies.

In terms of frequency, the corner dihedral is broadband and can perform for a wide-band of frequencies. On the other hand, the C-MIARM is narrow-band and can be sufficient at the given frequency of $14.7 \mathrm{GHz}$, as well as a tolerance of $150 \mathrm{MHz}$ around the selected frequency. This can be significant for applications such as automotive radar systems. However, for applications where broadband is needed, other type of unit cells ${ }^{31}$ can be proposed to ensure a stable distribution of the reflection phase at a wide-band of frequencies.

\section{EXPERIMENTAL MEASUREMENTS}

\section{A. $30^{\circ}$ Metasurface Prototype}

For the validation of of the numerical analysis of a $30^{\circ}$ metasurface, a prototype has been fabricated as shown in Fig. 5(b). with $7 \times 9$ periodic array of super-cells of dimensions $14.3 \mathrm{~cm} \times 9.2 \mathrm{~cm}$, printed on top of a Teflon grounded substrate of permittivity $\epsilon=2.2$ and thickness $1.6 \mathrm{~mm}$. The measurements were carried out in an anechoic chamber at an operating frequency of $14.7 \mathrm{GHz}$. The results have been compared to the simulation results as shown in Fig. 17.

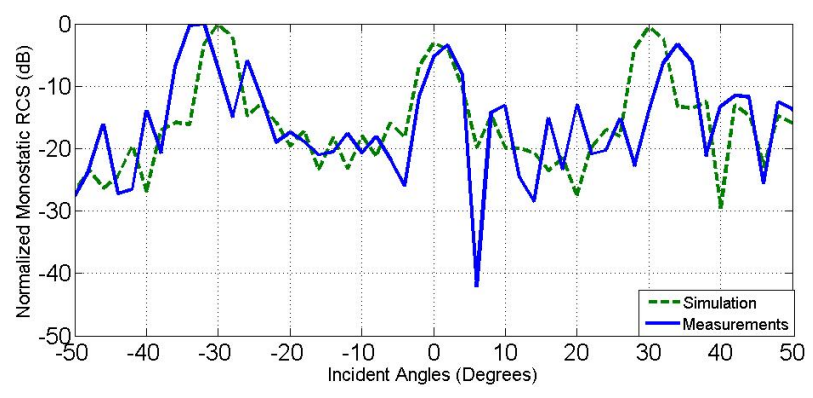

FIG. 17. Normalized monostatic RCS with respect to the incident angles of the experimental measurements compared to that of the simulation results for a $30^{\circ}$ metasurface prototype.

Fig. 17. shows that the measurement results are in good agreement with the numerical results.

\section{B. Experimental Measurements for the C-MIARM}

A prototype of the C-MIARM has been fabricated and tested as shown in Fig .18. The dimensions of the prototype are $8.163 \mathrm{~cm} \times 56.23 \mathrm{~cm}$. The measurements were carried out in an anechoic chamber using two Vivaldi antennas (transmitter and receiver) operating at $14.7 \mathrm{GHz}$. The measurements and simulation results have been compared as shown in Fig. 19.

The measurement results are said to follow and be in good agreement compared to that of the simulated re-

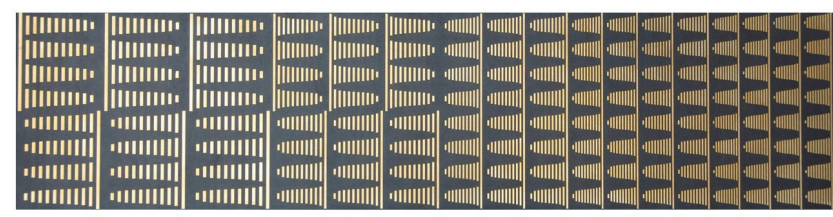

FIG. 18. Fabricated prototype of the C-MIARM of dimensions $8.163 \mathrm{~cm} \times 56.23 \mathrm{~cm}$.

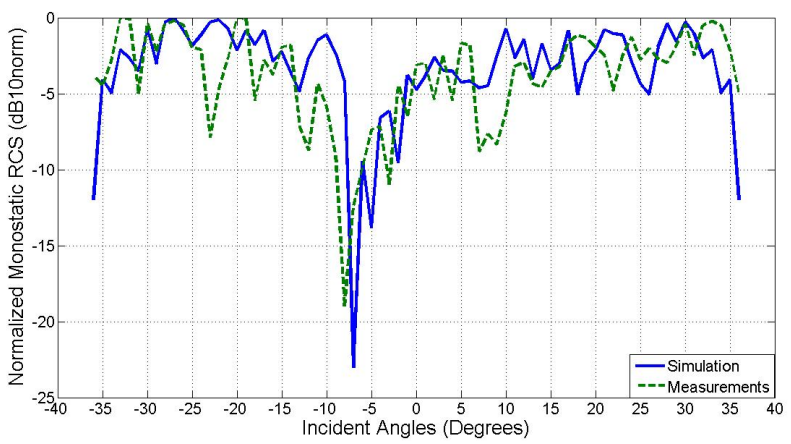

FIG. 19. Normalized Monostatic RCS with respect to the incident angles of the simulation results compared to that of the measurements at $14.7 \mathrm{GHz}$ for the C-MIARM.

sults with some losses at the angles of $8^{\circ}$ and $-23^{\circ}$ which can be due to mechanical inaccuracies, as well as the coupling and phase shift present between the two Vivaldi antennas, where a transceiver(radar system) would be the typical case for measurements of the monostatic RCS of the proposed prototype.

\section{DISCUSSION AND CONCLUSION}

A metasurface design has been proposed to achieve a retrodirective response at a wide range of incident angles. Different super-cell designs for various angles have been introduced based on the generalized phase law of reflection. It is observed that for a periodicity of higher than $2 \lambda$ or for designs for angles below $20^{\circ}$, the retroreflection loses its symmetrical response, i.e. retroreflection is no longer obtained identically for negative and positive values of the desired angle of incidence. A new design procedure was proposed using an opposite metasurface structure to recover the loss in the symmetrical direction. This design has been proposed to obtain a symmetrical response in both ranges between $-30^{\circ}$ to $-10^{\circ}$ and $10^{\circ}$ to $30^{\circ}$ for the C-MIARM. The obtained results for the proposed structure have shown to recover the loss in the reciprocal direction as well as a significant response for both ranges of the C-MIARM. Radar cross-section measurements for a metasurface of $30^{\circ}$ and the C-MIARM have been presented and shown to be good agreement with the numerical results.

The performance of the C-MIARM has been compared to the conventional corner dihedral, where a $50 \mathrm{~dB}$ gain has been noticed in the angular ranges $-30^{\circ}$ to $-20^{\circ}$ and $20^{\circ}$ to $30^{\circ}$ and similar performances between $-20^{\circ}$ to $-10^{\circ}$ 
and $10^{\circ}$ to $20^{\circ}$ compared to that of the corner dihedral which is limited between $-20^{\circ}$ to $20^{\circ}$. A $5 \mathrm{~dB}$ monostatic RCS has been obtained for a $8.2 \mathrm{~cm} \times 56.2 \mathrm{~cm}$ C-MIARM compared to that of a corner dihedral of dimensions $8 \mathrm{~cm} \times 15.5 \mathrm{~cm} \times 7.75 \mathrm{~cm}$. The advantage of the C-MIARM is having a 2D structure compared to the bulky 3-dimensional structure of the corner dihedral which can be difficult for implementation in different applications such as road applications

The C-MIARM can be a complement to existing topologies specially with its compact design and ability to address extreme incident angles. In future work, a more conformal design can be investigated to address wide range of incident angles for different applications.

\section{SUPPLEMENTARY MATERIAL}

See supplementary material for the complete dimensions of the super-cell designs at each angle of incidence and the phase of the reflection coefficient for each incident angle case.

\section{ACKNOWLEDGMENTS}

This work has been carried out in the context of the French national project CYCLOPE funded by Agence national de la recherche and the regional project ORIO in the framework of the ELSAT 2020 program co-financed by the European Union with the European Regional development fund, the French state and Hauts de France Regional council.

\footnotetext{
${ }^{1}$ H.-T. Chen, A. J. Taylor, and N. Yu, Reports on Progress in Physics 79, 076401 (2016).

${ }^{2}$ S. Sun, K.-Y. Yang, C.-M. Wang, T.-K. Juan, W. T. Chen, C. Y. Liao, Q. He, S. Xiao, W.-T. Kung, G.-Y. Guo, L. Zhou, and D. P. Tsai, Nano Letters 12, 6223 (2012), pMID: 23189928, http://dx.doi.org/10.1021/nl3032668.

${ }^{3}$ L. Zhang, S. Mei, K. Huang, and C.-W. Qiu, Advanced Optical Materials 4, 818 (2016).

${ }^{4}$ C. L. Holloway, E. F. Kuester, J. A. Gordon, J. O'Hara, J. Booth, and D. R. Smith, IEEE Antennas and Propagation Magazine 54, 10 (2012).

${ }^{5}$ A. A. Elsakka, V. S. Asadchy, I. A. Faniayeu, S. N. Tcvetkova, and S. A. Tretyakov, IEEE Transactions on Antennas and Propagation 64, 4266 (2016).
}

${ }^{6}$ A. Pors and S. I. Bozhevolnyi, Optics express 21, 27438 (2013). ${ }^{7}$ M. Pu, P. Chen, C. Wang, Y. Wang, Z. Zhao, C. Hu, C. Huang, and X. Luo, AIP advances 3, 052136 (2013).

${ }^{8}$ A. M. Wong and G. V. Eleftheriades, Physical Review X 8, 011036 (2018).

${ }^{9}$ Y. Huang, M. Pu, F. Zhang, J. Luo, X. Li, X. Ma, and X. Luo, Advanced Optical Materials, 1801480 (2019).

${ }^{10}$ A. Pors, M. G. Nielsen, R. L. Eriksen, and S. I. Bozhevolnyi, Nano letters 13, 829 (2013).

${ }^{11}$ Y. Li, J. Zhang, S. Qu, J. Wang, H. Chen, Z. Xu, and A. Zhang, Applied Physics Letters 104, 221110 (2014).

${ }^{12}$ W. T. Chen, K.-Y. Yang, C.-M. Wang, Y.-W. Huang, G. Sun, I.-D. Chiang, C. Y. Liao, W.-L. Hsu, H. T. Lin, S. Sun, et al., Nano letters 14, 225 (2013).

${ }^{13}$ L.-H. Gao, Q. Cheng, J. Yang, S.-J. Ma, J. Zhao, S. Liu, H.-B. Chen, Q. He, W.-X. Jiang, H.-F. Ma, et al., Light: Science \& Applications 4, 1 (2015).

${ }^{14}$ G. Zheng, H. Mühlenbernd, M. Kenney, G. Li, T. Zentgraf, and S. Zhang, Nature nanotechnology 10, 308 (2015).

${ }^{15}$ A. Ranjbar and A. Grbic, Physical Review B 95, 205114 (2017).

${ }^{16}$ S. Sun, Q. He, S. Xiao, Q. Xu, X. Li, and L. Zhou, Nature materials 11, 426 (2012).

${ }^{17}$ A. Pors, O. Albrektsen, I. P. Radko, and S. I. Bozhevolnyi, Scientific reports 3, 1 (2013).

${ }^{18}$ S. B. Glybovski, S. A. Tretyakov, P. A. Belov, Y. S. Kivshar, and C. R. Simovski, Physics Reports 634, 1 (2016).

${ }^{19}$ V. Fusco and N. Buchanan, IET Microwaves, Antennas \& Propagation $\mathbf{7}, 131$ (2013).

${ }^{20}$ A. Bunkowski, O. Burmeister, T. Clausnitzer, E.-B. Kley, A. Tünnermann, K. Danzmann, and R. Schnabel, Applied optics 45, 5795 (2006).

${ }^{21}$ N. Destouches, A. Tishchenko, J. Pommier, S. Reynaud, O. Parriaux, S. Tonchev, and M. A. Ahmed, Optics Express 13, 3230 (2005).

22 Z.-L. Deng, S. Zhang, and G. P. Wang, Nanoscale 8, 1588 (2016).

${ }^{23}$ E. G. Loewen, Applied optics and optical engineering. 9, 33 (1983).

${ }^{24}$ N. Yu, P. Genevet, M. A. Kats, F. Aieta, J.-P. Tetienne, F. Capasso, and Z. Gaburro, science 334, 333 (2011).

${ }^{25}$ E. Doumanis, G. Goussetis, G. Papageorgiou, V. Fusco, R. Cahill, and D. Linton, IEEE Transactions on Antennas and Propagation 61, 232 (2013).

${ }^{26}$ V. Asadchy, A. Díaz-Rubio, S. Tcvetkova, D.-H. Kwon, A. Elsakka, M. Albooyeh, and S. Tretyakov, Physical Review X 7, 031046 (2017).

${ }^{27}$ E. F. Knott, Radar cross section measurements (Springer Science \& Business Media, 2012).

${ }^{28}$ Y.-C. Guo, X.-W. Shi, and L. Chen, Progress In Electromagnetics Research B 5, 153 (2008).

${ }^{29}$ V. Asadchy, M. Albooyeh, S. Tcvetkova, A. Díaz-Rubio, Y. Ra'di, and S. Tretyakov, Physical Review B 94, 075142 (2016).

${ }^{30}$ A. Epstein and G. V. Eleftheriades, Physical Review B 90, 235127 (2014).

${ }^{31}$ Z. Chen, H. Deng, Q. Xiong, and C. Liu, Applied Physics A 124, 281 (2018). 